\title{
ALTERACIONES HEMATOLÓGICAS EN TRABAJADORES EXPUESTOS OCUPACIONALMENTE A MEZCLA DE BENCENO- TOLUENO-XILENO (BTX) EN UNA FÁBRICA DE PINTURAS
}

\author{
Luis Haro-García1,a, Nadia Vélez-Zamora²,b, Guadalupe Aguilar-Madrid²,c, Susana Guerrero-Rivera”, \\ Vanessa Sánchez-Escalante ${ }^{4, e}$, Sergio R. Muñoz ${ }^{5, f}$, Edward Mezones-Holguín $n^{6,7, g}$ \\ Cuauhtémoc Juárez-Pérez ${ }^{2, h}$
}

\begin{abstract}
RESUMEN
Objetivos. Evaluar las tres series celulares sanguíneas e identificar la presencia de hipocromía, macrocitosis, leucopenia, linfocitopenia y trombocitopenia en un grupo de trabajadores expuestos a la mezcla de benceno-tolueno-xileno (BTX). Materiales y métodos. Estudio transversal donde se incluyó a 97 trabajadores de una empresa de pinturas de México a los que se les realizó una biometría hemática convencional y les fue estimada la exposición a través de la dosis diaria potencial acumulada para vapores de BTX. Resultados. Del total de trabajadores, 19,6\%, mostró macrocitosis, $18,6 \%$, linfocitopenia, 10,3\% hipocromía, 7,2\% trombocitopenia y 5,2\% leucopenia. La asociación cruda de macrocitosis con exposición a dosis alta de mezcla de BTX fue la única significativa (OR:3,6; IC95\%: 1,08 - 13,9; $p=0,02$ ) y en la que se estructuró un modelo de regresión logística (OR:6,7; IC95\%: 1,33 - 13,55; p:0,02) ajustada por edad, consumo de alcohol y tabaquismo. Conclusiones. Todos los componentes citohemáticos analizados mostraron cambios leves; que podrían estar asociados con la exposición a la mezcla de BTX. De ellos, la macrocitosis podría constituirse en una manifestación precoz que merece ser vigilada.
\end{abstract}

Palabras clave: Enfermedades hematológicas; Trabajadores; Salud ocupacional; Derivados del benceno (fuente: DeCS BIREME).

\section{BLOOD DISORDERS AMONG WORKERS EXPOSED TO A MIXTURE OF BENZENE-TOLUENE-XYLENE (BTX) IN A PAINT FACTORY}

\begin{abstract}
Objectives. Evaluate the three blood cell series and identify the presence of hypochromia, macrocytosis, leucopenia, lymphopenia, and thrombocytopenia in a group of workers exposed to the mixture of benzene-toluene-xylene (BTX). Materials and methods. A cross-sectional study which included 97 workers from a paint factory in Mexico. The participants underwent conventional blood count and tests for potential cumulative daily dose of BTX fumes, to estimate exposure. Results. From the total of workers, $19.6 \%$ showed macrocytosis, $18.6 \%$, lymphopenia, hypochromia $10.3 \%, 7.2 \%$ and $5.2 \%$ thrombocytopenia leukopenia. The crude association of macrocytosis with exposure to high doses of BTX mixture was the only with statistical significance (OR: $3.6,95 \% \mathrm{Cl} 1.08$ to $13.9, \mathrm{P}=0.02$ ), and the base for a logistic regression model (OR: $6.7,95 \% \mathrm{Cl} 1.33$ to $13.55, \mathrm{P}=0.02$ ) adjusted for age, alcohol consumption, and smoking. Conclusions. All blood cytological components analyzed demonstrated mild changes, potentially associated with exposure to the mixture of BTX. Macrocytosis could constitute an early manifestation worthy for surveillance.
\end{abstract}

Key words: Hematologic diseases; Workers; Occupational health; Benzene derivatives (source: MeSH NLM).

\footnotetext{
Departamento de Salud Pública, Facultad de Medicina, Universidad Nacional Autónoma de México. México D.F., México.

Unidad de Investigación en Salud en el Trabajo, Centro Médico Nacional "Siglo XXI", Instituto Mexicano del Seguro Social. México D.F., México.

Servicio de Hematología, Hospital de Especialidades, Centro Médico Nacional “Siglo XXI”, Instituto Mexicano del Seguro Social. México D.F., México.

Departamento de Toxicología, Centro de Investigación y Estudios Avanzados, Instituto Politécnico Nacional. México D.F., México.

Centro de Excelencia CIGES, Facultad de Medicina, Universidad de la Frontera. Temuco, Chile.

Unidad de Generación y Análisis de Evidencias en Salud Pública, Instituto Nacional de Salud. Lima, Perú.

Escuela de Medicina, Universidad Peruana de Ciencias Aplicadas. Lima, Perú.

Médico especialista en Medicina Familiar y Salud en el Trabajo, doctor en Ciencias; ${ }^{b}$ ingeniera química maestra en Higiene Industrial; ${ }^{\mathrm{c}}$ médico especialista en Medicina del Trabajo, doctora en Ciencias; ${ }^{\mathrm{d}}$ médico especialista en Hematología, maestra en Ciencias; ${ }^{\mathrm{e}}$ médico especialista en Medicina del Trabajo; ${ }^{\mathrm{f}}$ matemático doctor en Filosofía en Bioestadística; ${ }^{\mathrm{g}}$ médico maestro en Ciencias en Epidemiología Clínica; ${ }^{\mathrm{h}}$ médico maestro en Ciencias en Salud Ambiental.
} 


\section{INTRODUCCIÓN}

La salud ocupacional es un área de relevancia en salud pública, no solo por el hecho de que las condiciones y desenlacesgeneradosafectandirectamentealapoblación económicamente activa, sino por sus consecuencias subsecuentes a nivel colectivo, en materia económica y social. En ese contexto, el estudio de los efectos de la exposición crónica a los compuestos aromáticos sobre los diferentes componentes de la médula ósea ha merecido un gran interés en la comunidad científica, debido fundamentalmente a su asociación con efectos citopénicos aun a concentraciones considerablemente bajas. Si bien, se ha investigado con mayor frecuencia los efectos dañinos producidos por el benceno (efecto leucemiogénico), en el mundo industrial moderno la exposición a esta sola sustancia no es frecuente; pues, el tolueno y el xileno son sus acompañantes regulares lo cual podría tener efectos aditivos que no han sido evaluados ${ }^{(1,2)}$.

Los metabolitos finales del benceno son responsables de los efectos perjudiciales en la médula ósea. La absorción de tolueno en forma separada ha mostrado efectos hematotóxicos poco claros, por lo que se ha considerado que la médula ósea no es órgano blanco de esta sustancia. Sin embargo, esta situación ha continuado bajo observación debido a reiterados resultados, aunque limitados y realizados solo en animales de experimentación, que asocian la exposición a tolueno con disminución de leucocitos, eritrocitos y plaquetas ${ }^{(2,3)}$.

Con relación al xileno, existe controversia sobre su efecto hematotóxico cuando se absorbe por inhalación. La exposición a isómeros de xileno ha evidenciado la formación de productos tóxicos intermedios y finales, semejantes a los del benceno, con efectos tóxicos en la médula ${ }^{(2-4)}$.

Por otro lado, los cambios hematológicos a determinarse como iniciales en personal expuesto a la mezcla de benceno-tolueno-xileno (BTX) son aún controvertidos ${ }^{(2)}$. Con relación a la fórmula blanca (FB), se ha observado quelacuentaleucocitaria disminuye en aproximadamente 1000 células $/ \mathrm{mm}^{3}$, entre los primeros cuatro meses de exposición a BTX, acompañada de linfocitopenia, mantenida considerada como el dato más precoz de hematotoxicidad ${ }^{(1,2)}$.

El primer cambio esperado en la fórmula roja (FR) es la disminución de la hemoglobina corpuscular media (HCM), la concentración media de hemoglobina corpuscular $(\mathrm{CMHC})$ y la presencia de macrocitosis ${ }^{(5)}$. Asimismo, no se ha demostrado que el resto de indicadores hematológicos de la FR sufran alteraciones importantes ${ }^{(5,6)}$. En fechas recientes se han iniciado investigaciones para conocer los efectos dañinos de los disolventes orgánicos en las plaquetas, proponiéndose que la trombocitopenia es el indicador de respuesta más sensible a la exposición de BTX ${ }^{(1,2)}$.

Para la comprensión integral del riesgo hematotóxico en trabajadores expuestos a BTX de manera crónica, se debe evaluar a las tres series celulares; es decir, la cuenta de glóbulos rojos con índices eritrocitarios, la cuenta total y diferencial de leucocitos y el número de plaquetas ${ }^{(2,3,5-7)}$. El objetivo de la presente investigación es evaluar las tres series celulares sanguíneas, e identificar la presencia de hipocromía, macrocitosis, leucopenia, linfocitopenia y trombocitopenia en un grupo de trabajadores de una fábrica de pinturas, expuestos a la mezcla de benceno-tolueno-xileno (BTX).

\section{MATERIALES Y MÉTODOS}

\section{DISEÑO Y POBLACIÓN DE ESTUDIO}

Se llevó a cabo un estudio transversal analítico sobre una muestra por conveniencia de 97 trabajadores de un total de 250 (fracción muestral de 38,8\%) de una fábrica de pinturas de México. Se identificaron trece áreas de trabajo: resinas, pintura base solvente, envasado de pintura base solvente, lacas, almacén de tambores, desarrollo de fórmulas, control de calidad, spitefire, almacén de producto terminado, pinturas emulsionadas, mantenimiento, secantes y almacén de polvos. Las primeras siete áreas se consideraron de alta exposición a BTX (los trabajadores cuentan y usan máscaras autofiltrantes para gases y vapores con arnés, compatible para uso de gafas y casco como equipo de protección personal); y las últimas seis de baja exposición a BTX ya que son áreas referidas básicamente como de producción de pintura base agua, de mantenimiento o almacenaje de pintura ya terminada, donde no se utiliza el equipo referido.

Se incluyeron trabajadores con antigüedad laboral no menor a cuatro meses continuos, sin discriminación por sexo ni puesto de trabajo. Fueron excluidos los trabajadores que declararon estar bajo tratamiento con medicamentos promotores de hematopoyesis; haber sido donante o receptor de sangre o hemoderivados por cualquier motivo, en los tres meses previos al estudio; estar en tratamiento con eritropoyetina o esteroides; padecer de púrpura trombocitopénica inmunológica; padecer enfermedad autoinmune o enfermedad infecciosa crónica tipo tuberculosis; tener síndrome de inmunodeficiencia adquirida, o hepatitis B o C. También se excluyeron a los trabajadores con cualquier proceso 
infeccioso agudo respiratorio o digestivo; a las mujeres que se encontraban en periodo de menstruación, y aquellos con epistaxis o hemorragia de consideración, a la fecha del estudio.

\section{VARIABLES, INSTRUMENTOS Y PROCEDIMIENTOS}

A través de un cuestionario autoaplicado se recolectaron los datos sobre edad, antigüedad laboral, antecedentes de tabaquismo, consumo de alcohol y uso de inhalantes.

Previo consentimiento informado, de la vena radial o cubital se extrajeron $5 \mathrm{~mL}$ de sangre de cada trabajador, muestras que se conservaron en tubos con $5 \mathrm{~g}$ de ácido etilendiaminotetracético (EDTA). Las mediciones cuantitativas y los índices eritrocitarios, FB y cuenta de plaquetas se llevaron a cabo en el Laboratorio de Análisis Clínicos del Hospital de Oncología del Centro Médico Nacional "Siglo XXI" del Instituto Mexicano del Seguro Social según el manual de procedimientos de la institución. Se determinó hipocromía (CMHC $\leq 31,5 \mathrm{~g}$ $\mathrm{Hb} / \mathrm{dL}$ ), macrocitosis (VCM $\geq 95 \mathrm{fL}$ ), leucopenia ( $\leq 4500$ leucocitos $/ \mathrm{mm}^{3}$ ), linfocitopenia (<1500 linfocitos $/ \mathrm{mm}^{3}$ ) y plaquetopenia (<149 000 plaquetas) ${ }^{(8)}$

La concentración de exposición $\left(\mathrm{C}_{\mathrm{E}}\right)$ se determinó a partir de la medición del aire a la altura de la zona respiratoria a través de bombas que permitieron el muestreo de los vapores a flujos menores de $500 \mathrm{~mL} /$ minuto y como medio de captura a tubos de carbón activado de cáscara de coco de $6 \mathrm{~mm}$ de diámetro por $70 \mathrm{~mm}$ de longitud con secciones de 50/100 mg de material adsorbente. Las bombas se calibraron antes e inmediatamente después del muestreo en el campo.

El tiempo base para el muestreo fue la jornada de ocho horas las cuales se cubrieron con cuatro o cinco muestras recolectadas de forma continua, con cálculo para cada una de ellas del flujo de operación de la bomba y el tiempo de duración del muestreo.

Para el control de calidad del proceso de muestreo se utilizaron blancos de campo que acompañaron a las muestras hasta su destino final para ser analizadas. Las muestras obtenidas se enviaron al Laboratorio de Salud en el Trabajo del Instituto Mexicano del Seguro Social, con sede en la ciudad de Orizaba, Veracruz, México, donde se empleó para su análisis un cromatógrafo de gases marca Thermoquest Trace GC 2000 ${ }^{\circledR}$ con detector de ionización de flama o columnas capilares y empacadas para la separación de BTX.

Las dos secciones de los tubos de carbón fueron analizadas por separado y cada una de ellas por duplicado, como parte del procedimiento de control de calidad analítico; utilizando, además, estándares certificados trazables a NIST (National Institute for Standars and Technology) de cada una de las sustancias, para su identificación y cuantificación.

Con base en la $C_{E}$, se estimó la exposición por vía aérea de vapores de BTX de los 97 trabajadores por ruta inhalatoria y se propuso como la sumatoria de la dosis diaria potencial acumulada $\left(\sum \mathrm{D}_{\mathrm{DPA}}\right)$ de vapores de cada uno de los componentes de dicha mezcla y cuyos efectos se están considerando como aditivos, o que actúen en el mismo órgano blanco; cada una de las $D_{D P A}$ representa la cantidad de vapores de cada uno de los disolventes contenidos en el aire inspirado y acumulado durante la jornada, de tal manera que $D_{D P A}=\left(E_{A J}\right)\left(V_{A l}\right)$ donde $E_{A J}$ es la suma acumulada de las concentraciones encontradas en exposiciones parciales, considerando una jornada de trabajo $\left(E_{A J}=\sum E_{\text {parcial }}\right.$ en $\mathrm{mg} / \mathrm{m}^{3}$ por jornada) y $V_{A l}$ es el volumen de aire inspirado en $\mathrm{m}^{3}$. El estimador se estableció también de acuerdo al número de meses laborados en la empresa por cada trabajador.

\section{ANÁLISIS ESTADÍSTICO}

Se generó una base de datos en el paquete estadístico STATA versión 10.0 (STATA Corp, TXT, USA) Se llevó a cabo un análisis descriptivo utilizando medidas de tendencia central y de dispersión (media y desviación estándar) para las variables numéricas y frecuencias absolutas y relativas para el caso de las variables categóricas.

Asimismo, se efectuó un análisis crudo para evaluar la asociación entre las alteraciones hematológicas (macrocitosis, hipocromía, leucopenia, linfocitopenia, y trombocitopenia) o la presencia de, al menos, una de ellas, con la dosis potencial acumulada de exposición a la mezcla de BTX alta (>652,3 mg de BTX/kg/ jornada/años laborados) y baja ( $\leq 652,3 \mathrm{mg}$ de BTX/ $\mathrm{kg} /$ jornada/años laborados), con cálculo de la razón de odds (OR) e intervalos de confianza al 95\% (IC95\%). Adicionalmente, se evaluó la asociación entre cada una de las variables de respuesta propuestas con cada una de las variables que se controlaron: edad del trabajador, antigüedad laboral, antecedente de tabaquismo, consumo de alcohol, uso de inhalantes y área de trabajo donde se desempeña. A este nivel también se utilizó pruebas de significancia estadística; en ese sentido, se utilizó la prueba t de student para evaluar si existen diferencias entre los valores de variables numéricas cuando se compararon por una categórica (con o sin alteración hematológica) y test exacto de Fisher en el caso de la evaluación de la asociación entre dos variables categóricas. 
Finalmente, se constituyó un modelo con regresión logística binaria no condicional, con cálculo de OR, para cada una de las alteraciones hematológicas propuestas, con inclusión de las respectivas interacciones de estas variables con la dosis potencial acumulada de exposición a mezcla de BTX. En todas los modelos propuestos se llevaron a cabo medidas globales de bondad de ajuste con prueba $\mathrm{X}^{2}$ de Pearson y razón de verosimilitud.

\section{ASPECTOS ÉTICOS}

El presente estudio fue aprobado por el Subcomité de Ética del Comité Nacional de Investigación del Instituto Mexicano del Seguro Social. Fue registrado con el número IMSS/PROT-140/2004.

\section{RESULTADOS}

\section{DESCRIPCIÓN DE LA POBLACIÓN}

La edad promedio de los participantes fue de 34,6 \pm 8,1 años (rango: 19 a 54), con una mediana de antigüedad laboral en la empresa de 48 meses (rango: 6 a300); el $45 \%$ ha laborado 55 meses por lo menos sin cambiar de puesto de trabajo. El 91\% consume alcohol en forma rutinaria y el $76 \%$ presentó el antecedente de consumo de tabaco.

\section{VALORES HEMATOLÓGICOS}

Los valores promedios encontrados fueron: $6,92 \times 10^{3} \pm$ $1,6 / \mathrm{mm}^{3}$ de leucocitos; $5,3 \times 10^{6} \pm 0,4 / \mathrm{mm}^{3}$ de eritrocitos; $230 \times 10^{3} \pm 54,1 / \mathrm{mm}^{3}$ de plaquetas; $16,1 \pm 1,2 \mathrm{~g}$ de hemoglobina/dL; 89,9 \pm 7,1 fL/eritrocito de VCM; 30,1 \pm 1,5 pg/eritrocito de $\mathrm{HCM}$ y $33,2 \pm 1,3 / \mathrm{dL}$ de eritrocitos de CMHC; 4306, $9 \pm 1466 / \mathrm{mm}^{3}$ de neutrófilos; $1947 \pm 570 /$ $\mathrm{mm}^{3}$ de linfocitos, y $344,5 \pm 190 / \mathrm{mm}^{3}$ de monocitos. Se encontró hipocromía en el $10,3 \%$ de los participantes; macrocitosis en el 19,6\%; leucopenia en el 5,2\%; linfocitopenia en el 18,6\%, y plaquetopenia en el 7,2\%. Se encontró, al menos, una de estas cinco alteraciones hematológicas en el $48,5 \%$ de todas las muestras procesadas.

Se encontraron diferencias significativas al comparar la presencia de hipocromía, macrocitosis o, al menos, una alteración hematológica (de las cinco evaluadas) en relación con la edad y la antigüedad laboral (Tabla 1). Los casos positivos tuvieron, en promedio, mayor edad y antigüedad laboral que los casos negativos. El valor promedio de VCM fue el único que particularmente presentó diferencia significativa entre trabajadores con antigüedad mayor o igual a 55 meses $(91,6 \pm 6,6 \mathrm{fL})$ frente a los que tuvieron menos antigüedad $(88,65 \pm 7,2 \mathrm{fL})$.
Tabla 1. Alteraciones hematológicas por la exposición a BTX según la edad y antigüedad laboral.

\begin{tabular}{lcccc}
\hline & $\begin{array}{c}\text { Edad en } \\
\text { años } \\
\text { (media DE) }\end{array}$ & $\mathbf{p}$ & $\begin{array}{c}\text { Antigüedad } \\
\text { laboral en meses } \\
\text { (media DE) }\end{array}$ & $\mathbf{p}$ \\
\hline Hipocromía & & & & \\
\hline Sí (n=10) & $40,7 \pm 9,5$ & 0,01 & $151,7 \pm 10,4$ & 0,02 \\
NO (n=87) & $40,7 \pm 9,5$ & & $80,9 \pm 8,9$ & \\
Macrocitosis & & & & \\
Sí ( $n=19)$ & $39,2 \pm 9,2$ & $<0,05$ & $125,6 \pm 9,5$ & 0,05 \\
NO (n=78) & $33,5 \pm 7,4$ & & $79,2 \pm 9,0$ & \\
$\begin{array}{l}\text { Al menos una } \\
\text { alteración }\end{array}$ & & & & \\
hematológica & & & & \\
Sí ( $n=47)$ & $36,4 \pm 8,3$ & $<0,05$ & $103 \pm 9,7$ & 0,05 \\
NO (n=50) & $33,0 \pm 7,6$ & & $73,8 \pm 8,6$ & \\
\hline
\end{tabular}

$\mathrm{DE}$ : desviación estándar.

\section{BTX EN EL AMBIENTE DE TRABAJO}

La mediana de la dosis diaria potencial acumulada para mezcla de vapores de BTX por jornada laboral de ocho horas fue de 15,46 mg/kg (rango: 1,31-344,11); la mediana de esta dosis según antigüedad en la empresa fue de 652,3 mg/kg (rango: 25,7 - 82586,4), la cual se estableció como punto de corte para determinar exposición "alta" o "baja" a la mezcla de disolventes propuesto. De acuerdo con ello, el 49,5\% de los trabajadores están sujetos a una alta exposición de BTX.

Tabla 2. Análisis crudo entre las alteraciones hematológicas y la exposición a BTX.

\begin{tabular}{|c|c|c|c|}
\hline $\begin{array}{c}\text { Alteración } \\
\text { hematológica }\end{array}$ & $\begin{array}{c}\text { Expuestos a } \\
\text { dosis alta de } \\
\text { BTX } \\
\text { (> 652,3 mg) }\end{array}$ & $\begin{array}{c}\text { Expuestos a } \\
\text { dosis baja de } \\
\text { BTX } \\
(\leq 652,3 \mathrm{mg})\end{array}$ & OR (IC95\%) \\
\hline \multicolumn{4}{|l|}{ Hipocromía } \\
\hline sí & 6 & 4 & $1,6(0,3-8,2)$ \\
\hline NO & 42 & 45 & \\
\hline \multicolumn{4}{|l|}{ Macrocitosis } \\
\hline sí & 14 & 5 & $3,6(1,1-13,9)$ \\
\hline NO & 34 & 44 & \\
\hline \multicolumn{4}{|l|}{ Leucopenia } \\
\hline sí & 2 & 3 & $0,6(0,0-6,1)$ \\
\hline NO & 46 & 46 & \\
\hline \multicolumn{4}{|l|}{ Linfocitopenia } \\
\hline sí & 9 & 9 & $1,0(0,3-3,2)$ \\
\hline NO & 39 & 40 & \\
\hline \multicolumn{4}{|l|}{ Plaquetopenia } \\
\hline sí & 1 & 6 & $0,1(0,0-1,3)$ \\
\hline NO & 47 & 43 & \\
\hline \multicolumn{4}{|l|}{$\begin{array}{l}\text { Al menos una } \\
\text { Alteración }\end{array}$} \\
\hline sí & 27 & 20 & $1,8(0,7-4,5)$ \\
\hline $\mathrm{NO}$ & 21 & 29 & \\
\hline
\end{tabular}


Tabla 3. Análisis de las manifestaciones hematológicas y las variables de control.

\begin{tabular}{|c|c|c|c|c|c|c|}
\hline \multirow[t]{2}{*}{$\begin{array}{c}\text { Alteración } \\
\text { hematológica * }\end{array}$} & \multicolumn{6}{|c|}{ Variables de control OR (IC95\%) } \\
\hline & Edad $>35$ años & $\begin{array}{c}\text { Antigüedad en } \\
\text { la empresa > } 55 \\
\text { meses }\end{array}$ & $\begin{array}{l}\text { Ingesta de } \\
\text { alcohol }\end{array}$ & Tabaquismo & Uso de drogas & $\begin{array}{l}\text { Área de trabajo } \\
\text { con exposición } \\
\text { alta a BTX }\end{array}$ \\
\hline \multirow[t]{2}{*}{ Hipocromía } & 3,6 & 3,1 & 1,3 & 0,7 & 0,2 & 1,0 \\
\hline & $(0,7-22,9)$ & $(0,6-19,9)$ & $(0,2-65,8)$ & $(0,1-4,5)$ & $(0,0-2,3)$ & $(0,2-5,2)$ \\
\hline \multirow[t]{2}{*}{ Macrocitosis } & 2,8 & 4,5 & 2,1 & 1,8 & 1,01 & 0,5 \\
\hline & $(0,9-9,6)$ & $(1,3-17,2)$ & $(0,2-96,2)$ & $(0,4-10,8)$ & $(0,3-3,5)$ & $(0,2-1,7)$ \\
\hline \multirow[t]{2}{*}{ Leucopenia } & 2,1 & 0,8 & 0,4 & 2,1 & 1,9 & 1,0 \\
\hline & $(0,2-26,4)$ & $(0,1-7,2)$ & $(0,0-21,1)$ & $(0,2-98,4)$ & $(0,2-18,2)$ & $(0,1-12,6)$ \\
\hline \multirow[t]{2}{*}{ Linfocitopenia } & 0,8 & 0,5 & 0,7 & 0,8 & 0,8 & 0,3 \\
\hline & $(0,2-2,6)$ & $(0,2-1,8)$ & $(0,1-8,3)$ & $(0,2-3,1)$ & $(0,7-2,8)$ & $(0,1-0,9)$ \\
\hline \multirow[t]{2}{*}{ Plaquetopenia } & 1,0 & 0,2 & 0,9 & 0,8 & 1,1 & 0,5 \\
\hline & $(0,1-6,4)$ & $(0,0-1,6)$ & $(0,1-47,5)$ & $(0,1-8,5)$ & $(0,1-7,6)$ & $(0,1-3,0)$ \\
\hline \multirow[t]{2}{*}{$\begin{array}{l}\text { Al menos una } \\
\text { alteración }\end{array}$} & 2,0 & 1,5 & 2,0 & 1,0 & 0,7 & 0,3 \\
\hline & $(0,8-4,9)$ & $(0,6-3,7)$ & $(0,4-13,0)$ & $(0,4-2,9)$ & $(0,3-2,1)$ & $(0,1-0,9)$ \\
\hline
\end{tabular}

* OR de referencia: sin presencia de la alteración hematológica.

Los resultados de las asociaciones crudas y promedios de la dosis diaria potencial acumulada para mezcla de BTX se presentan en la Tabla 2. La asociación de las variables de control y las diferentes manifestaciones hematológicas identificadas se presentan en la Tabla 3. Al realizar una regresión logística, se encontró asociación de macrocitosis con la dosis potencial acumulada de exposición a BTX (OR: 6,77 IC95\%: 1,33 - 13,55 p=0,02) ajustada por edad > 35 años, consumo de alcohol y tabaquismo.

\section{DISCUSIÓN}

Aunque las formas identificadas se consideraron como muy leves, todos los componentes citohemáticos analizados mostraron cambios que podrían estar asociados con la exposición a BTX. Sin embargo, no mostraron diferencias con las que presentan los trabajadores cuya antigüedad en la empresa fue menor a los 55 meses, la cual ha sido señalada como el lapso de exposición ocupacional, al menos a la fracción bencénica que, de mantenerse de manera sostenida, explicaría dichos cambios $^{(1)}$; ello no quiere decir que la antigüedad en el puesto de trabajo no intervenga en el riesgo hematotóxico en estos trabajadores; lo que sí es posible señalar es la necesidad de revisar, y posiblemente modificar, la antigüedad en el puesto de trabajo a partir de la cual el efecto hematotóxico sea referido como sostenible ${ }^{(6,9,10)}$; por otro lado, la frecuencia de consumo de alcohol y tabaquismo de la población trabajadora estudiada es considerable y de algún modo estas variables podrían estar también involucradas para explicar los cambios de referencia ${ }^{(11,12)}$.

Cabe señalar que los valores utilizados para categorizar la presencia o no de dichas manifestaciones hematológicas fueron extraordinariamente rígidos, a tal grado que podría llegarse a considerar que estas no poseen ningún significado estrictamente clínico y, por ello, su relevancia es disminuida; de hecho, se ha señalado que los valores obtenidos a través de estudios convencionales pueden considerarse como espurios, como podría ser el caso de los datos presentados ${ }^{(2,13)}$. Sin embargo, la importancia parece radicar más en su vigilancia y observación a través del tiempo, que del valor específico de una sola observación puntual de un estudio convencional de biometría hemática practicado a un trabajador en lo particular ${ }^{(14)}$. La existencia de medios más precisos para la medición de los diferentes parámetros citohematológicos no debería invalidar la utilidad de la biometría hemática convencional, la cual es una forma relativamente económica e inocua a partir de la cual se podría decidir efectuar mediciones más precisas o emitir medidas preventivas más prácticas a favor de los trabajadores expuestos de manera crónica ${ }^{(1,2,15-19)}$.

Dentro de las alteraciones hematológicas identificadas se vio macrocitosis, la cual puede deberse a la exposición crónica al benceno que produce cambios estructurales de todos los componentes celulares en la médula ósea; además de su capacidad anaplásica y leucemiogénica. Asimismo, aunque se considera que el tolueno y el xileno en forma individual no parecen tener 
efectos hematotóxicos importantes, estos sí podrían aparecer al exponerse a la mezcla de estas sustancias e incluso existe la posibilidad de potencializar los efectos dañinos cuando coexiste el benceno, aun a dosis extremadamente bajas. Cabe añadir que la presencia de macrocitosis también se ha relacionado con la ingesta consuetudinaria de alcohol, característica frecuente en la población del estudio ${ }^{(15,20-24)}$. Otra alteración hallada fue la hipocromía, que también podría ser atribuida al elevado número de participantes que afirmaron fumar $\mathrm{y}$ beber en forma rutinaria ${ }^{(17,25)}$.

Dentro de las limitaciones, señalaremos que como es común en investigaciones realizadas en centros de trabajo de países en vías de desarrollo, el estudio se fundamentó en los trabajadores que dispuso la empresa para ser sujetos a análisis; esto se refleja en la potencia estadística al no contar con una muestra calculada, que, al mismo tiempo, tampoco fue aleatoria, y el no contarse básicamente con suficientes recursos para incluir un grupo comparable de trabajadores no expuestos a BTX ${ }^{(28)}$. Asimismo, es importante reconocer que en algunos casos no se pudo haber encontrado asociación estadísticamente significativa por motivo de que la potencia estadística no haya sido suficiente, esto debido a que no se efectuó un cálculo de tamaño de muestra ad hoc.

Finalmente, concluimos que todos los componentes citohemáticos analizados mostraron cambios, aunque muy leves, que podrían estar asociados con la exposición crónica de la mezcla de BTX. A través de una biometría hemática convencional es posible identificar datos cardinales que sugieren posibles efectos hematotóxicos (macrocitosis e hipocromía), por lo que se recomienda considerar estos parámetros en las evaluaciones de rutina en este tipo de trabajadores. Adicionalmente, se sugiere llevar a cabo nuevas investigaciones que incorporen muestreos representativos con mayor cantidad de sujetos de investigación.

\section{Contribuciones de autoría}

LHG: Concepción y diseño del estudio. NV: medición de los vapores, análisis en el Laboratorio de Salud en el Trabajo, redacción de los resultados. GAM: diseño del estudio, análisis e interpretación de la información, redacción de resultados. SGR: revisión del proyecto, análisis e interpretación de los resultados hematológicos, revisión crítica. VSE: redacción del borrador y análisis de datos, apoyo en la redacción final. SRM: diseño de estudio, análisis estadístico, redacción de resultados, revisión crítica del manuscrito; EMH: redacción del manuscrito, revisión del análisis estadístico, revisión crítica del manuscrito. CJP: supervisión de la medición de vapores de BTX, búsqueda de financiamiento, revisión crítica del manuscrito. Todos los autores dieron su aprobación final del manuscrito.

\section{Fuentes de financiamiento}

CONACYT (Consejo Nacional de Ciencia y Tecnología de México) Salud 4004-C01-77.

\section{Conflictos de interés}

Los autores declaran no tener conflictos de interés en la publicación de este artículo.

\section{REFERENCIAS BIBLIOGRÁFICAS}

1. U.S. Department of Health and Human Services. Toxicological Profile for Benzene. Atlanta, GA: Agency for Toxic Substances and Disease Registry (ATSDR); 1993.

2. U.S. Department of Health and Human Services. Interaction profile for: Benzene, Toluene, Ethylbenzene, and Xylenes (BTEX). Atlanta, GA: Agency for Toxic Substances and Disease Registry (ATSDR); 2004.

3. Moszczynski P, Lisiewicz J. Hematological indicators of peripheral blood in workers occupationally exposed to benzene, toluene and xylene. Folia Med Cracov. 1984;25(34):405-19.

4. D’Azevedo PA, Tannhauser M, Tannhauser SL, Barros HM. Hematological alterations in rats from xylene and benzene. Vet Hum Toxicol. 1996;38(5):340-4.

5. Qu Q, Shore R, Li G, Jin X, Chen LC, Cohen B, et al. Hematological changes among Chinese workers with a broad range of benzene exposures. Am J Ind Med. 2002;42(4):275-85.

6. Hancock DG, Moffitt AE Jr, Hay EB. Hematological findings among workers exposed to benzene at a coke oven by-product recovery facility. Arch Environ Health. 1984;39(6):414-8.

7. Chan CC, Shie RH, Chang TY, Tsai DH. Workers' exposures and potential health risks to air toxics in a petrochemical complex assessed by improved methodology. Int Arch Occup Environ Health. 2006;79(2):135-42.

8. McKenzie SB. Hematología Clínica, 2a Ed. México: Manual Moderno; 2000.

9. Van Raalte HG, Grasso P. Hematological, myelotoxic, clastogenic, carcinogenic, and leukemogenic effects of benzene. Regul Toxicol Pharmacol. 1982;2(2):153-76.

10. Ruiz MA, Vassallo J, de Souza CA. [Hematologic changes in patients chronically exposed to benzene]. [Article in Portuguese]. Rev Saude Publica. 1993;27(2):145-51.

11. Baarson KA, Snyder CA. Evidence for the disruption of the bone marrow microenvironment by combined exposures to inhaled benzene and ingested ethanol. Arch Toxicol. 1991;65(5):414-20.

12. Saita G. [Hematological damage of occupational origin in industry]. [Article in Italian]. Med Lav. 1978;69 Suppl 3(3 Suppl):350-5.

13. Schnatter RA, Kerzic PJ, Zhou Y, Chen M, Nicolich MJ, Lavelle K, et al. Peripheral blood effects in benzeneexposed workers. Chem Biol Interact. 2010;184(1-2):174-81.

14. Rothman N, Smith MT, Hayes RB, Li GL, Irons RD, Dosemeci M, et al. An epidemiologic study of early biologic effects of benzene in Chinese workers. Environ Health Perspect. 1996;104 Suppl 6:1365-70. 
15. Beving $\mathbf{H}$, Tornling $\mathbf{G}$, Olsson $\mathbf{P}$. Increased erythrocyte volume in car repair painters and car mechanics. $\mathrm{Br} \mathrm{J}$ Industr Med. 1991;48(7):499-501.

16. Swaen GM, van Amelsvoort L, Twisk JJ, Verstraeten E, Slootweg R, Collins JJ, et al. Low level occupational benzene exposure and hematological parameters. Chem Biol Interact. 2010;184(1-2):94-100.

17. Hristeva-Mirtcheva V. Changes in the peripheral blood of workers with occupational exposure to aromatic hydrocarbons. Int Arch Occup Environ Health. 1998;71 Suppl:S81-3.

18. Collins JJ, Conner P, Friedlander BR, Easterday PA, Nair RS, Braun J. A study of the hematologic effects of chronic low-level exposure to benzene. J Occup Med. 1991;33(5):619-26.

19. Jacobs A, Geary C, Osman J. Haematological disorders and occupational hazards: a British Society for Haematology/Health and Safety Executive study. $\mathrm{Br} \mathrm{J}$ Haematol. 1993;84(3):555-7.

20. Kaferle J, Strzoda CE. Evaluation of macrocitosis. Am Fam Physician. 2009;79(3):203-8.

21. Rumsey SE, Hokin B, Magin PJ, Pond D. Macrocytosis-an Australian general practice perspective. Aust Fam Physician. 2007;36(7):571-2.

22. Aslinia F, Mazza J, Yale H. Megaloblastic anemia and other causes of macrocytosis. Clin Med Res. 2006;4(3)23541. Erratum in: Clin Med Res. 2006 Dec;4(4):342.

23. Davidson RJL, Hamilton PJ. High mean red cell volume: its incidence and significance in routine haematology. J Clin Pathol. 1978;31(5):493-8.
24. Haro-García L, González-Bonilla CR, Chacón-Salinas R, Pérez-Lucio C, Juárez-Pérez CA, Borja-Aburto VH. Manifestaciones hemato/inmunológicas asociadas a exposición ocupacional a mezcla de BTX (benceno-toluenoxileno). Rev Med Inst Mex Seguro Soc. 2008;46(6):64350 .

25. Aksoy M, Dinçol K, Akgün T, Erdem S, Dinçol G. Haematological effects of chronic benzene poisoning in 217 workers. Br J Ind Med. 1971;28(3):296-302.

26. Chen Z, Liu SJ, Cai SX, Yao YM, Yin H, Ukai H, et al. Exposure of workers to a mixture of toluene and Xylene. II Effects. Occup Environ Med. 1994;51:47-9.

27. Wallén M, Holm S, Nordqvist MB. Coexposure to toluene and $\mathrm{p}$-xylene in man: uptake and elimination. $\mathrm{Br} \mathrm{J}$ Ind Med. $1985 ; 42(2): 111-6$

28. Nuwayhid IA. Occupational health research in developing countries: a partner for social justice. Am J Public Health. 2004;94(11)1916-21.

Correspondencia: Luis Cuauhtémoc Haro García Dirección: Av. Cuauhtémoc 330, Edificio "C", 1er. Piso, Delegación Cuauhtémoc, 06725, México, D.F. Teléfono: (+15) 56276900 anexo: 21661 Correo electrónico: luisharo2@hotmail.com

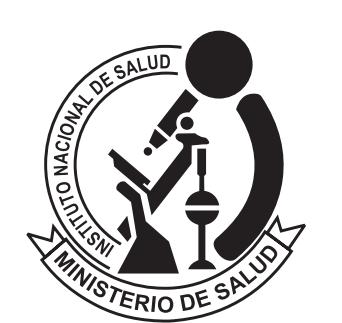

Investigar para proteger la saluo

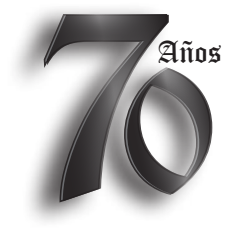

REVISTA PERUANA DE MEDICINA

EXPERIMENTAL Y SALUD PÚBLICA

CUMPLIENDO SUS METAS Y

PROYECTÁNDOSE AL FUTURO

Visite los contenidos de la revista en: www.ins.gob.pe/rpmesp 